Document downloaded from:

http://hdl.handle.net/10251/156113

This paper must be cited as:

Galindo, J.; Navarro, R.; García-Cuevas González, LM.; Tarí, D.; Tartoussi, H.; Guilain, S. (2019). A zonal approach for estimating pressure ratio at compressor extreme off-design conditions. International Journal of Engine Research. 20(4):393-404.

https://doi.org/10.1177/1468087418754899

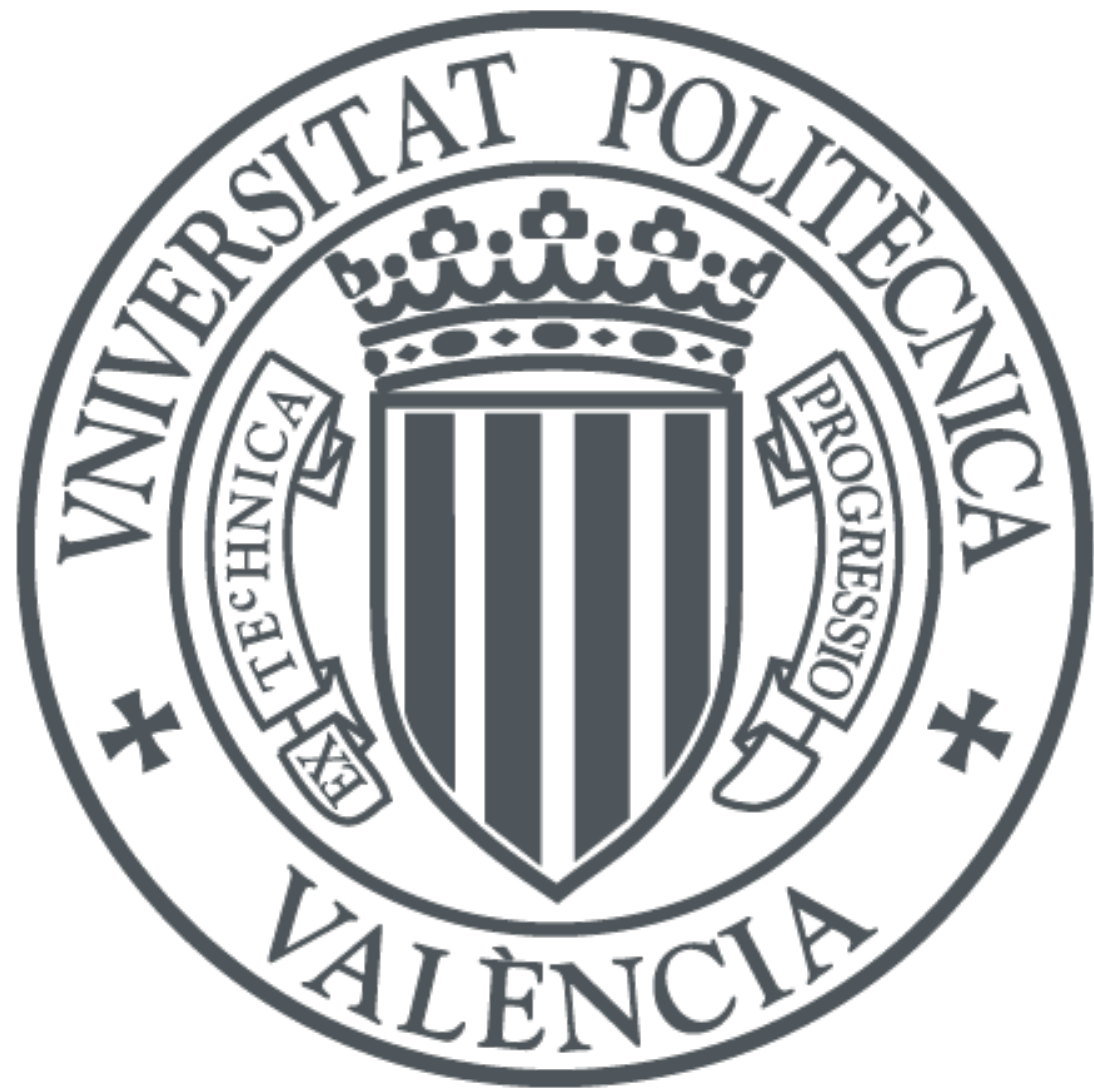

The final publication is available at

https://doi.org/10.1177/1468087418754899

Copyright SAGE Publications

Additional Information

This is the author s version of a work that was accepted for publication in International Journal of Engine Research. Changes resulting from the publishing process, such as peer review, editing, corrections, structural formatting, and other quality control mechanisms may not be reflected in this document. Changes may have been made to this work since it was submitted for publication. A definitive version was subsequently published as https://doi.org/10.1177/1468087418754899 


\section{NOTICE:}

this is the author's version of a work that was accepted for publication in International Journal of Engine Research. Changes resulting from the publishing process, such as peer review, editing, corrections, structural formatting, and other quality control mechanisms may not be reflected in this document. Changes may have been made to this work since it was submitted for publication. A definitive version was subsequently published as:

\section{References}

[1] J. Galindo, R. Navarro, L. M. García-Cuevas, D. Tarí, H. Tartoussi, S. Guilain, A zonal approach for estimating pressure ratio at compressor extreme off-design conditions, International Journal of Engine Research doi:10.1177/1468087418754899. 


\title{
A zonal approach for estimating pressure ratio at compressor extreme off-design conditions
}

\author{
J. Galindo ${ }^{\mathrm{a}}$, R. Navarro ${ }^{\mathrm{a}}$, L.M. García-Cuevas ${ }^{\mathrm{a}}$, D. Taría ${ }^{\mathrm{a}}$, H. Tartoussi ${ }^{\mathrm{b}}, \mathrm{S}$. \\ Guilain $^{\mathrm{b}}$ \\ ${ }^{a}$ CMT - Motores Térmicos, Universitat Politècnica de València \\ Camino de Vera, 46022 Valencia \\ ${ }^{b}$ Renault, FR
}

\begin{abstract}
0D-1D computational fluid dynamics (CFD) codes are used to simulate the performance of complete internal combustion engines. In such codes, the operation of a turbocharger compressor is usually addressed employing its performance map. However, simulation of engine transients may drive the compressor to work at operating conditions outside the region provided by the manufacturer map. Therefore, a method is required to extrapolate the performance map to extended off-design conditions. This work examines several extrapolating methods at the different off-design regions, namely, low pressure ratio zone, low speed zone and high speed zone. The accuracy of the methods is assessed with the aid of compressor extreme off-design measurements. In this way, the best method is selected for each region and the manufacturer map is used in design conditions, resulting in a zonal extrapolating approach aiming to preserve accuracy. The transitions between extrapolated zones are corrected, avoiding discontinuities and instabilities.
\end{abstract}

Keywords: Compressor map, compression ratio, off-design extrapolation, mathematical model

Email addresses: galindo@mot.upv.es (J. Galindo), ronagar1@mot.upv.es (R. Navarro), luiga12@mot.upv.es (L.M. García-Cuevas), datade@mot.upv.es (D. Tarí), hadi.tartousi@renault.com (H. Tartoussi), stephane.guilain@renault.com (S. Guilain) 


\section{Introduction}

As pollutant regulations for internal combustion engines (ICE) are becoming more restrictive year by year, manufacturers are forced to design, certify and construct new engines as fast as possible. This rush, combined with the high amount of independent variables and parameters present in the engine and its subsystems, impels the design stages to rely on gas dynamic codes such as GT-Power [1, 2] or OpenWAM,[3, 4] since modern computational resources are powerful enough to run numerous simulations in a short period of time. Thus, the improvement of the codes to assure accuracy and convergence of their simulations is critical. When a turbocharged ICE needs to be simulated, the most widespread compressor submodels in gas dynamic simulations are based on the use of a compressor map where the operating condition is interpolated from corrected mass flow and speed.

Compressor off-design conditions have been thoroughly studied experimentally and numerically. [5, 6, 7, 8] However, less research has been devoted to the analysis of extreme off-design points outside the standard corrected maps. Experimentally, it is hard to measure extreme off-design conditions due to different reasons depending on the region of the map, although some authors have been able to characterize extended maps.[9, 10] In Fig. 1 it is depicted a generic compressor map expressed in terms of corrected mass flow $\left(\dot{m}^{*}\right)$, compression ratio $\left(\Pi_{c}\right)$ and corrected compressor iso-speed lines $(\mathrm{N})$, indicating the different extreme off-design regions.

At low compressor speeds (LS) the sensitivity of the flow-meter is too low to provide accurate measurements. Conducting experiments at low pressure ratio region (LPR) requires an enhanced test rig, as explained in section 3 . Moreover, in this region oil leaks due to the unbalanced axial forces between the compressor and the turbine.[11, 12] The low isentropic efficiency of the compressor in this zone is the ultimate reason for manufacturers to avoid conducting measurements at low pressure ratios. Poor efficiency is explained by the high mass flow rates, which generate important pressure losses due to friction and compressibility effects. In fact, at certain points choke appears when the flow velocity approaches the speed of sound. Once the wheel is completely blocked at sonic conditions, the corrected mass flow cannot raise further and the compression ratio suddenly drops, as observed in the isospeed lines of the compressor map. Finally, at high speeds (HS) the moving components reach their safety mechanical limit, so that measuring beyond a certain compressor speed is avoided to preserve the turbocharger integrity. 


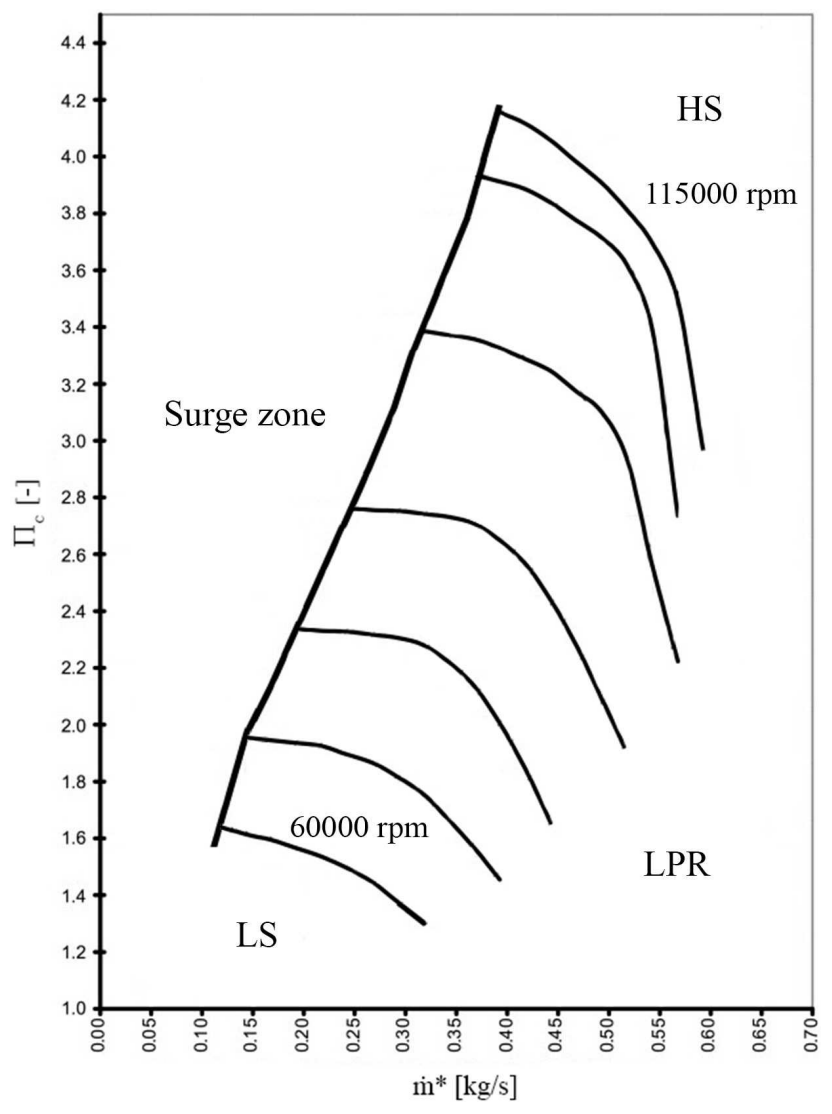

Figure 1: Example of compressor map, indicating the extreme off-design regions.

Sometimes, during the simulation of the 1D dynamic model, the operating point of the compressor goes into one of these extreme off-design regions. This can happen during the initial convergence phase, due to a low load engine operation or even in a transient evolution. [13, 14] It is important for a proper calculation to be able to have information in extreme off-design conditions, avoiding discontinuities that could lead to errors. This work is addressed to provide a model that allows extending a common compressor map range to assure the accuracy and convergence of the engine cycle simulation. The issues to measure off-design operating points have been already described, but modelling these conditions is challenging as well: at lowest speeds, the problem is the quick inception into turbine operating mode, and thus the short operating range. The blockage needs to model adequately the losses in the static part of the stage and the surge region is natural unsteady and 
unstable. At highest compressor speeds, the model should deal with severe compressibility effects, which affect the work exchange and the losses.

Due to the multiple and complex phenomena found in the different offdesign regions, it is difficult to address the extrapolation with a physicallybased modelling approach. Moreover, a single extrapolation fitting method may not be able to provide a good performance in all of these areas. Thus, the extrapolation approach described in this paper proposes to treat each zone separately, taking advantage of the benefits of the most suitable extrapolation method for each region. This paper is focused in developing a strategy for extending the pressure ratio map, which is eventually combined with the method to extrapolate isentropic efficiency described by Galindo et al. [15] so that the 1D code is able to represent the compressor performance at all potential working conditions.

Next section will cover the literature review performed regarding the extrapolation issue, describing how other authors dealt with it. The experimental apparatus and the compressor maps used for validating the model are introduced in section 3. Section 4 is devoted to describe the proposed extrapolation approach, comparing different models found in the literature at each region and selecting the most accurate. Finally, in section 5 two additional compressor maps are used to test the proposed approach and the concluding remarks are provided in section 6 .

\section{Literature background}

\subsection{Jensen-based models}

One of the most extended extrapolation method applied to compressor maps is the Jensen's model.[16] This method proposes a relation between the flow coefficient $(\phi)$, the load coefficient $(\psi)$ and the inlet tip-speed Mach number $\left(M_{1}\right)$ defined in Eqs. (1), (2) and (3).

$$
\begin{gathered}
\psi=\frac{\left(\Pi_{c}^{\frac{\gamma-1}{\gamma}}-1\right) C_{p} T_{1}}{\frac{1}{2} U_{2}^{2}} \\
\phi=\frac{\dot{m}_{c}}{\frac{\pi}{4} d_{c}^{2} U_{2} \rho_{1}} \\
M_{1}=\frac{U_{1}}{\sqrt{\gamma R T_{1}}}
\end{gathered}
$$


Together with the efficiency, the performance of the compressor is completely defined.[17] The relation between the dimensionless coefficients proposed by Jensen, defined in Eq. (4), is controlled with six adjustable parameters that are calibrated with a least squares method using all the available points in the measured compressor map.

$$
\psi=\frac{k_{1}+k_{2} M_{1}+k_{3} \phi+k_{4} M_{1} \phi}{k_{5}+k_{6} M_{1}-\phi}
$$

This adjustment allows the extrapolation of the map to higher and lower compressor speeds since the rotating velocity is included in the definition of the equation. Generally, reasonable results are obtained with this method at low speeds. Jensen et al. [16] considered that the coefficients of Eq. (4) should remain constant and equal to the coefficients of the lowest speed for extrapolating below the lowest measured iso-speed line. This assumption is correct for near-incompressible flows, since the iso-speed lines collapse when plotted as flow and load coefficients. Indeed, the convenient behaviour of flow and load coefficients is employed not only for compressor map extrapolation but for interpolation within iso-speed lines as well.[18] However, the definition of Eq. (4) is unable to capture the shape of the iso-speed lines within the choke region.

Based on the Jensen's proposal, other authors took advantage of the same flow to load coefficient relation but introducing modifications that improve the extrapolation accuracy at high compressor speed. Dowell and Akehurst [19] proposed adjusting Eq. (4) for each iso-speed line, eliminating the dependence of the compressor speed and simplifying the equation, becoming Eq. (5).

$$
\psi=\frac{k_{1_{i}}+k_{2_{i}} \phi}{k_{3_{i}}-\phi}
$$

At this moment, the whole compressor map is defined with three coefficients per iso-speed line. These coefficients can be modeled applying a polynomial regression as a function of the compressor speed allowing the extrapolation at high and low compressor speeds.

Another approach for the Jensen method was carried out by Martin et al. [20] and used by El Hadef.[21] Martin et al. also used the simplified non-isospeed-dependant Jensen's equation for adjusting the compressor map. For interpolation in this approach, the coefficients are defined in a linear piecewise function between each iso-speed line. 
To extrapolate at low speeds, Martin et al. take advantage of the parabolic shape of the maximum efficiency curve and the isentropic enthalpy rise, which can be tuned so that the maximum efficiency of the extrapolated iso-speed line coincides with the parabolic maximum efficiency curve. This model will be referred in section 4 simply as "Martin" for the sake of simplicity.

\subsection{Müller's model}

Müller et al. [22] proposed a correlation of the compression ratio and the corrected mass flow by using the isentropic enthalpy rise. Eqs. (6) and (7) define this relation.

$$
\begin{gathered}
\Pi_{c}=\left(\frac{\Delta h_{i s}}{c_{p} T_{1}}+1\right)^{\frac{\gamma}{\gamma-1}} \\
\Delta h_{i s}=s_{1} U_{2}^{2}+s_{2} \dot{m}^{* 2}+s_{3} \dot{m}^{*}+s_{4}
\end{gathered}
$$

Four adjustable parameters are tuned to fit with measured data. The main drawback of this approach is the poor prediction of the choke phenomenon, meaning huge error at high compressor speed. At low speed, where the flow behaves more incompressibly, the adjustment is better resulting in a realistic solution.

\subsection{Leufvén's model}

Leufvén et al. $[9,23,24,25]$ employed a generalized-ellipse definition to fit the compressor map. Eq. (8) defines the ellipse function based on eleven adjustable parameters corresponding to coefficients appearing in Eqs. (9)(13), which are calibrated with the measured compressor map. This method allows low and high speed extrapolation and is able to conform fully choked flow behaviour. Again, this model will be named in section 4 simply as "Leufvén" for word economy.

$$
\begin{gathered}
\Pi=C_{5}\left(1-\left(\frac{\dot{m}^{*}-C_{4}}{C_{3}-C_{4}}\right)^{C_{1}}\right)^{\frac{1}{C_{2}}} \\
C_{1}=C_{10}+C_{11} N^{*} \\
C_{2}=C_{20}+C_{11} N^{* C_{22}} \\
C_{3}=C_{30}+C_{31} N^{*} \\
C_{4}=C_{41} N^{* C_{42}} \\
C_{5}=1+C_{51} N^{* C_{52}}
\end{gathered}
$$




\section{Experimental campaign}

\subsection{Experimental method}

Extreme off-design conditions are unable to be reached with an engine test bench, since the measured turbocharger map would inherit the engine operating constraints. Therefore, a gas stand rig is employed. Furthermore, LPR points (see Fig. 1) are difficult to measure in normal turbocharger gas stands. The maximum flow rate at each speed line is constrained by the back-pressure of the compressor outlet line, which produces maps that are limited far away from choked flow. An special setup was used to obtain the compressor maps used in this work.

The gas stand scheme, which is described by Serrano et al.,[10] is shown in 2. The gas generator used for the turbine in this gas stand is a truck engine. This engine is braked with a reversible asynchronous motor, so it can also be powered to work with cold gas flowing through the turbine in order to minimise the heat transfer inside the turbocharger. The engine discharges towards a plenum (a): it is connected to the turbine and the exhaust line, so the total flow of the turbine can be controlled regardless of the rotational speed of the engine by means of two automatic valves (1). In these experiment, the turbine is used to drive the compressor at the required rotational speed.

A normal compressor map is measured controlling its rotational speed and its loading by means of the turbine and two electrical valves (4) that are installed in parallel. To measure extended maps, the compressor inlet can be pressurised and force-fed. The asynchronous motor is also connected to a gearbox that drives an auxiliary screw compressor, which is used for boosting the engine and the turbocharger compressor inlet. The level of boosting pressure can be controlled by the rotational speed of the engine that drives the screw compressor and by the combination of two automatic valves 2 and (3). As lower levels of compressor pressure ratio are being measured, valve (3) is opened and the rotational speed of the engine is increased.

There are measurement stations in the compressor inlet and outlet lines, as well as in the turbine inlet and outlet lines and in the engine intake. The mass flow, pressure and temperature are measured in these points. The temperatures are measured by means of 4 type-K thermocouples per measurement station. Piezorresistive transducers are employed to register the different pressure levels. The compressor and engine flow rates are measured with thermal flowmeters, whereas the turbine outlet flow is measured 
by means of a differential-pressure based flowmeter. The rotational speed is measured with an inductive sensor. The facility has an independent lubrication system for the turbocharger, as well as an independent cooling system. The latter was not employed during these experiments, however, as they were performed under cold turbine flow conditions. [26] Alternatively, a postprocessing of the measurements with an adiabatizing model $[27,11]$ could have been used. The expanded uncertainty of the main compressor operating variables, computed as described in the work carried out by Olmeda et al.,[28] is shown in 1. It is computed using a coverage factor of 3 for a $99.7 \%$ confidence (assuming normal distributions) .

\begin{tabular}{|l|l|l|}
\hline Sensor & Type & Uncertainty \\
\hline Flow rate & $\begin{array}{l}\text { Direct measure- } \\
\text { ment, thermal } \\
\text { flow meter }\end{array}$ & $\begin{array}{l}1 \% \text { of the } \\
\text { measure- } \\
\text { ment }\end{array}$ \\
\hline $\begin{array}{l}\text { Inlet pres- } \\
\text { sure }\end{array}$ & $\begin{array}{l}\text { Direct measure- } \\
\text { ment, 2 piezorre- } \\
\text { sistive }\end{array}$ & $6 \mathrm{hPa}$ \\
\hline $\begin{array}{l}\text { Outlet } \\
\text { pressure }\end{array}$ & $\begin{array}{l}\text { Direct measure- } \\
\text { ment, 2 piezorre- } \\
\text { sistive }\end{array}$ & $15 \mathrm{hPa}$ \\
\hline $\begin{array}{l}\text { Inlet tem- } \\
\text { perature }\end{array}$ & $\begin{array}{l}\text { Direct measure- } \\
\text { ment, 4 type-K } \\
\text { thermocouples }\end{array}$ & $0.8 \mathrm{~K}$ \\
\hline $\begin{array}{l}\text { Corrected } \\
\text { mass flow }\end{array}$ & Derived & $\begin{array}{l}1.4 \% \\
\text { the value } \\
\text { (max.) }\end{array}$ \\
\hline $\begin{array}{l}\text { Pressure } \\
\text { ratio }\end{array}$ & Derived & $\begin{array}{l}0.01 \\
\text { (max.) }\end{array}$ \\
\hline
\end{tabular}

Table 1: Expanded uncertainties of the compressor working variables

\subsection{Tested compressors}

Two compressor maps, depicted in Figs. 3(a) and 3(b), are used to assess the ability of the different models to reproduce extreme off-design conditions. Notice that compressor A does not achieve fully choked conditions, i.e. vertical iso-speed lines, what will gain importance during the extrapolation of the higher compressor speed. 


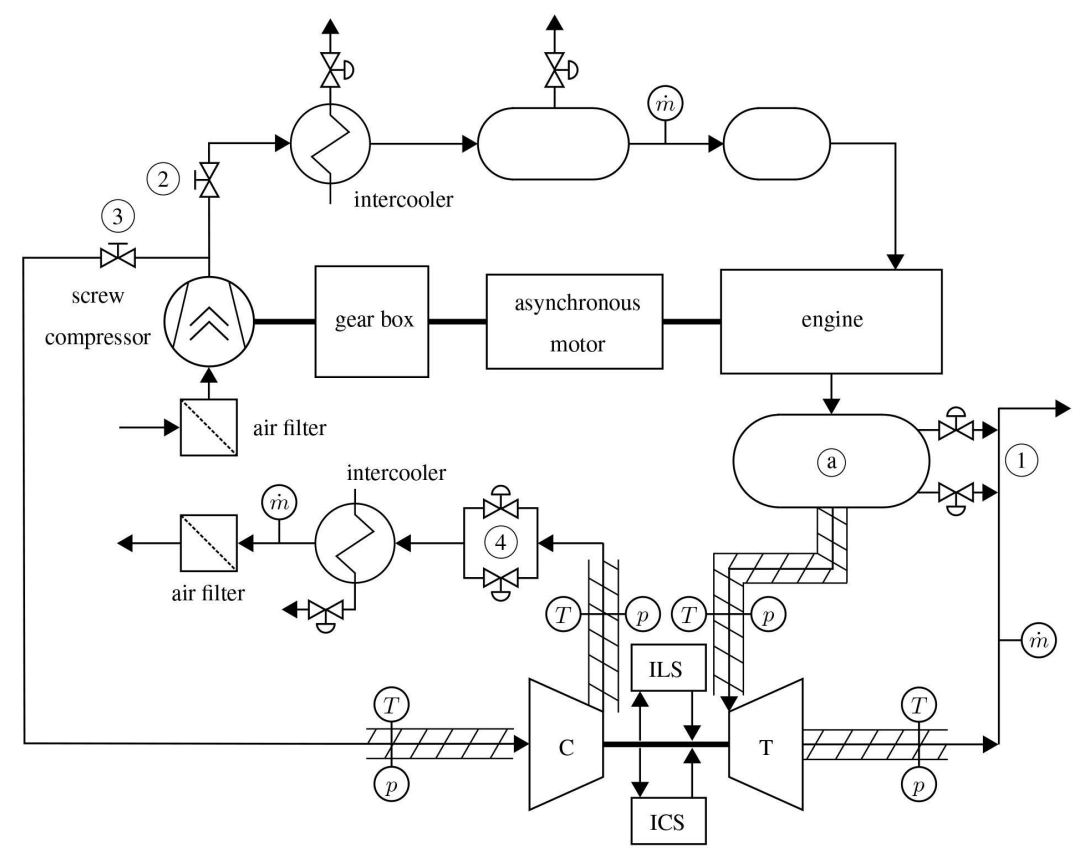

Figure 2: Gas stand scheme

For the validation of the model, extreme off-design points of the maps ought to be removed in order to simulate the characteristics of a common compressor map. As the proposed approach consists in a zonal procedure, where each separated region is extrapolated independently, different sets of points are considered as input. In first place, the low pressure ratio region is extrapolated. In this case, points with efficiencies below $50 \%$ are removed (below the solid red lines of Fig. 3). Next, for the low speed extrapolation, in addition to the removal of low efficiency points, speed lines with a tip-speed Mach number below 0.7 are taken out and the extrapolation is conducted to predict them (below the dashed red lines of Fig. 3). Finally, for the high speed extrapolation, the low efficiency points and the highest speed line are removed as well. The extrapolation should be able then to reproduce this particular high speed line (above the dotted red lines of Fig. 3).

\section{Proposed extrapolation approach}

This section describes the developed extrapolation method, which employs a combination of the models described in section 2 together with some 




(a) Compressor $\mathrm{A}$

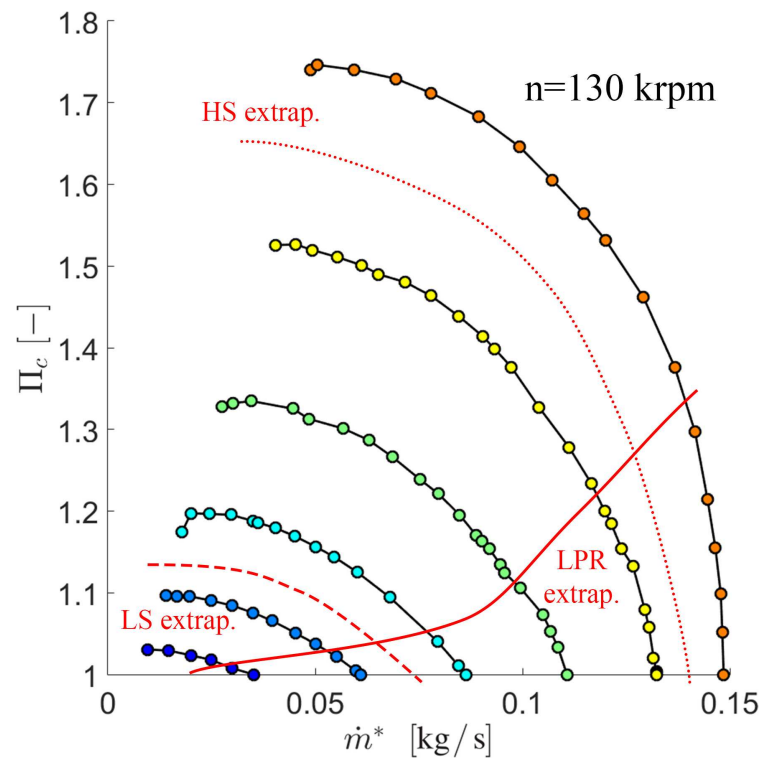

(b) Compressor B

Figure 3: Compressor maps including extreme off-design measurements

enhancements to increase accuracy. 


\subsection{Pre-processing}

Before the extrapolation procedure is applied, data processing must be carried out to properly prepare the sample of measured points to be used.

\subsubsection{Removal of redundant points}

Sometimes, measurements are performed in very-close operating points. This produces redundancy manifested in an undesired steep change in the slope of the iso-speed line. The use of splines to connect the data is quite widespread, i.e., a curve is forced to cross every single point corresponding to a certain iso-speed. Therefore, if there are two consecutive points very close, the curve may reproduce spurious local maximums or minimums. Due to these potential issues, the points with a relative difference in corrected mass flow rate below a fixed criteria ( $\sim 5 \%$ of average separation) are removed from the database. An example of redundant point can be found in the highest iso-speed line in Fig. 4.

\subsubsection{Removal of positive slope region}

Some methods consist in fitting a curve defined with an equation with adjustable parameters that cannot get the shape of a positive slope close to the surge region. Therefore, to avoid accuracy losses, those points are removed from the database.

In Fig. 4 it is depicted the compressor B map after the pre-processing treatment. Inside the circles are highlighted the points that were removed from the database during this process. It is noticeable that the positive slope region of two iso-speed lines and a redundant point have been marked to be removed.

\subsection{Low pressure ratio extrapolation}

The first region to be extrapolated by the model is the low pressure ratio. This is because the majority of methods rely on extending the iso-speed lines, including additional extrapolation features in order to generate new iso-speed lines. As the simplest process to do this is just to use the measured data as a way to extend the speed lines, this will be the first step of the current model. Of the above explained methods found in the literature, the Martin's model [20] and the ellipse model developed by Leufvén [23] are compared.

Figs. 5(a) and 5(b) show the comparison between Martin's and Leufvén's models. The most critical point of this extrapolation, the mass flow at $\Pi_{c}=$ 1 , is used to calculate the error of each model. A summary of the comparison 


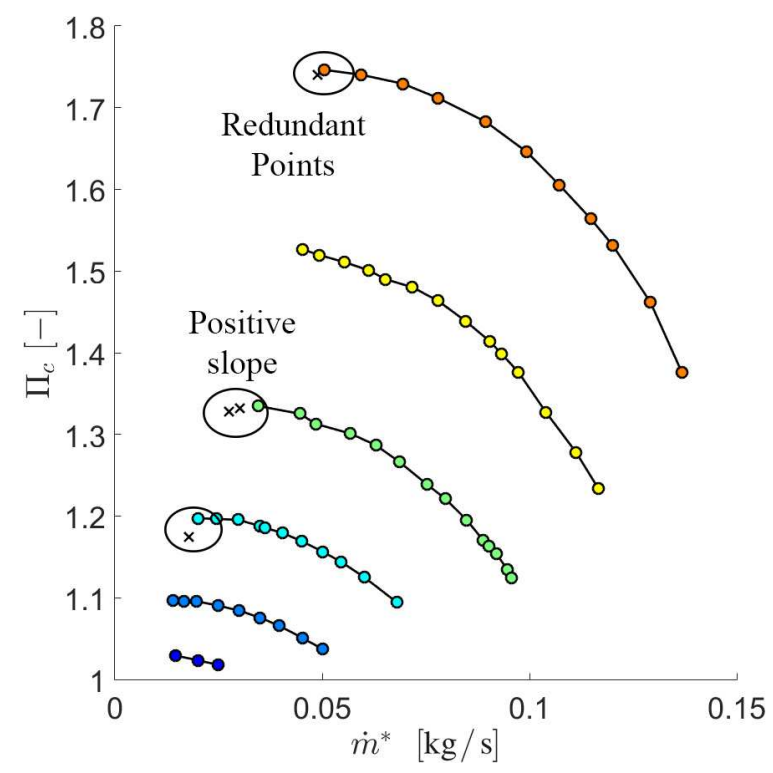

Figure 4: Map B pointing out the operating points to be removed by the pre-processing procedure

between these models and the measured data is shown in Table 2. In this case, Leufvén's model produced an error between 1 and $4 \%$ whilst Martin's was less accurate providing an error between 4 and $8 \%$. These errors grow as the compressor speed increases since the choke phenomenon is hard to predict. The ellipse method, in addition of extrapolating with greater accuracy, is consistent since one equation is used for the whole range creating dependence between the different lines. This implies the iso-speed lines do not cross between themselves but instead follow a smooth tendency. Therefore, it is decided to select this model for extrapolating this region in the developed approach. In order to merge the extrapolated region with the measured map, a linear blending is performed, as described in section 4.5.1.

\subsection{Low speed extrapolation}

The possibility to extrapolate to other compressor speeds with Leufvén's method [23] is straightforward once the constants are tuned with the measured map. For a low speed extrapolation using a Jensen-based model it is decided to adjust the coefficients of the simplified version of the Jensen's equation (Eq. 5) with only the lowest iso-speed line available (considering in addition its low pressure ratio extrapolation calculated previously). Then, 


\begin{tabular}{|l|c|c|c|c|}
\hline & Comp.A & Comp.A & Comp.B & Comp.B \\
\hline Iso-speed & $\epsilon$ Martin & $\epsilon$ Leufvén & $\epsilon$ Martin & $\epsilon$ Leufvén \\
\hline$[\#]$ & {$[\%]$} & {$[\%]$} & {$[\%]$} & {$[\%]$} \\
\hline $\mathbf{1}(\mathrm{N} \downarrow)$ & 11.80 & 1.31 & 2.93 & 8.25 \\
\hline $\mathbf{2}$ & 15.35 & 0.10 & 4.87 & 2.31 \\
\hline $\mathbf{3}$ & 10.11 & 1.24 & 8.22 & 0.64 \\
\hline $\mathbf{4}$ & 3.78 & 1.98 & 3.48 & 0.44 \\
\hline $\mathbf{5}$ & 4.27 & 1.13 & 1.35 & 3.13 \\
\hline $\mathbf{6}$ & 7.72 & 1.30 & 4.10 & 7.11 \\
\hline $\mathbf{7}$ & 7.68 & 1.53 & - & - \\
\hline $\mathbf{8}(\mathrm{N} \uparrow)$ & 2.67 & 1.10 & - & - \\
\hline averg. & 7.92 & 1.21 & 4.16 & 3.65 \\
\hline
\end{tabular}

Table 2: Low pressure ratio extrapolation comparison (error in mass flow rate at $\pi_{c}=1$ ).

keeping constant those coefficients, lower speed lines are generated. The influence of the compressor speed is produced controlling the blade tip speed $(U)$ in the flow coefficient equation (Eq. 2).

The extrapolation of the low speed lines is shown for compressor $\mathrm{A}$ in Fig. 6(a) and for compressor B in Fig. 6(b). For calculating the error of each model, the compression ratio is taken in the first place. The mean error of all possible points in terms of compression ratio is calculated: the summary of this comparison is shown in Table 3. Then, the error of the corrected mass flow at $\Pi_{c}=1$ is calculated as well: the comparison of these errors is shown in Table 4. Leufvén model correctly predicts the shape of the extrapolated line in compressor A. Nevertheless, Jensen's equation model is more accurate specially in compressor $\mathrm{B}$ in which the errors in terms of compression ratio are around $7 \%$ while with Leufvén's error increases up to 20\%. Concerning the error of the corrected mass flow at $\Pi_{c}=1$, Jensen's equation model achieves values below $2 \%$ while Leufvén's model is less accurate as can be observed in Fig. 6(b), resulting in errors close to 16\%. As Jensen's equation model has demonstrated accuracy extrapolating at low speeds, it is going to be selected for the final approach of this work. Additional features needed for this region to be used in a 1D gas dynamic code compressor map, such as surge line extrapolation and number of extrapolated lines, are exposed in sections 4.5.2 and 4.5.4. 


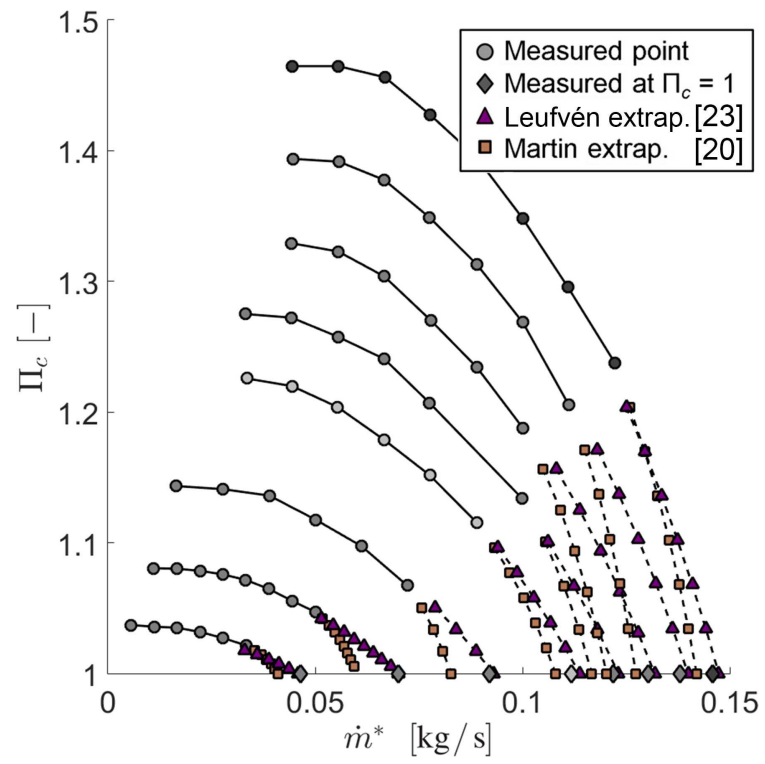

(a) Compressor $\mathrm{A}$

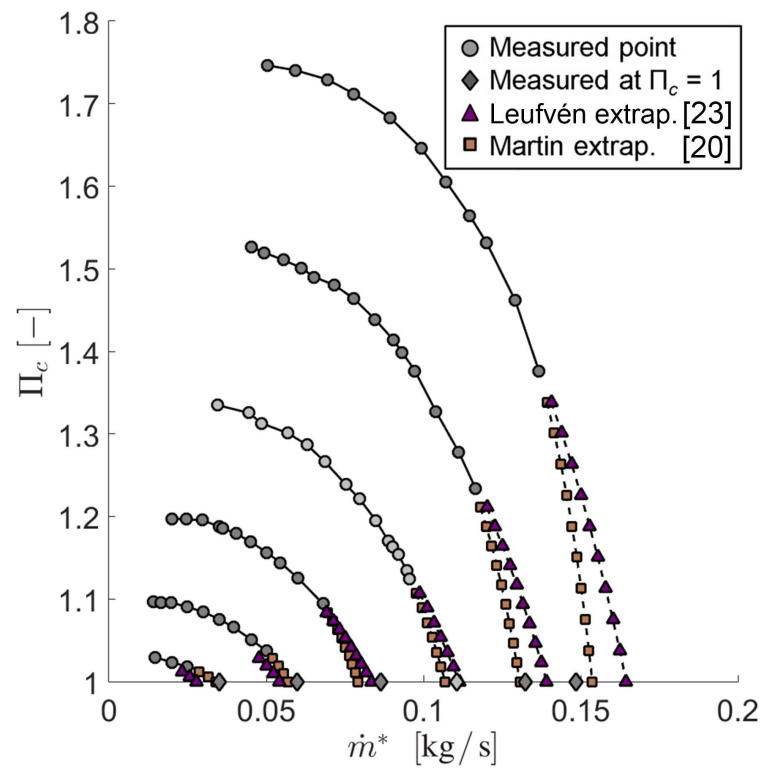

(b) Compressor B

Figure 5: Comparison of LPR-extrapolating methods

\subsection{High speed extrapolation}

For this final region of extrapolation, only Leufvén's model [23] is compared with the measured data since Martin's model does not provide a sat- 


\begin{tabular}{|l|c|c|c|c|}
\hline & Comp.A & Comp.A & Comp.B & Comp.B \\
\hline Iso-speed & $\epsilon$ Jensen & $\epsilon$ Leufvén & $\epsilon$ Jensen & $\epsilon$ Leufvén \\
\hline$[\#]$ & {$[\%]$} & {$[\%]$} & {$[\%]$} & {$[\%]$} \\
\hline $\mathbf{1}(\mathrm{N} \downarrow)$ & 5.84 & 15.83 & 7.55 & 14.86 \\
\hline $\mathbf{2}$ & 4.92 & 7.87 & 5.65 & 34.18 \\
\hline $\mathbf{3}(\mathrm{N} \uparrow)$ & 6.37 & 3.32 & - & - \\
\hline averg. & 5.71 & 9.01 & 6.60 & 24.52 \\
\hline
\end{tabular}

Table 3: Low speed extrapolation comparison (error in compression ratio).

\begin{tabular}{|l|c|c|c|c|}
\hline & Comp.A & Comp.A & Comp.B & Comp.B \\
\hline Iso-speed & $\epsilon$ Jensen & $\epsilon$ Leufvén & $\epsilon$ Jensen & $\epsilon$ Leufvén \\
\hline$[\#]$ & {$[\%]$} & {$[\%]$} & {$[\%]$} & {$[\%]$} \\
\hline $\mathbf{1}(\mathrm{N} \downarrow)$ & 2.08 & 1.98 & 0.73 & 20.96 \\
\hline $\mathbf{2}$ & 2.65 & 0.02 & 1.68 & 9.64 \\
\hline $\mathbf{3}(\mathrm{N} \uparrow)$ & 1.07 & 1.45 & - & - \\
\hline averg. & 1.93 & 1.15 & 1.20 & 15.30 \\
\hline
\end{tabular}

Table 4: Low speed extrapolation comparison (error in mass flow rate at $\pi_{c}=1$ ).

isfactory high speed extrapolation approach.[20] In addition, as it is hard to predict the behaviour of the compressor performance at this region (very high compressor speeds and choke appearance), difficulties appear when extrapolating at even higher speeds. The extrapolation using Leufvén's method is direct, nevertheless special attention must be paid over the positive slope region of the line. This method can not predict positive slopes, so that this part has to be extrapolated independently (see section 4.5.3). The error of Leufvén's model is calculated following the same definition used in the previous steps, is based on the corrected mass flow at $\Pi_{c}=1$ and it is shown in table 5 .

Figs. 7(a) and 7(b) show the high speed extrapolation, where the error of Leufvén's model is found to be around 1\% for compressor A and around $7 \%$ for compressor B. The extrapolation of compressor A is accurate, however the extrapolation of the high speed line of compressor $\mathrm{B}$ results to be quite accurate for high compression ratios but introducing the error when approaching $\Pi_{c}=1$. 


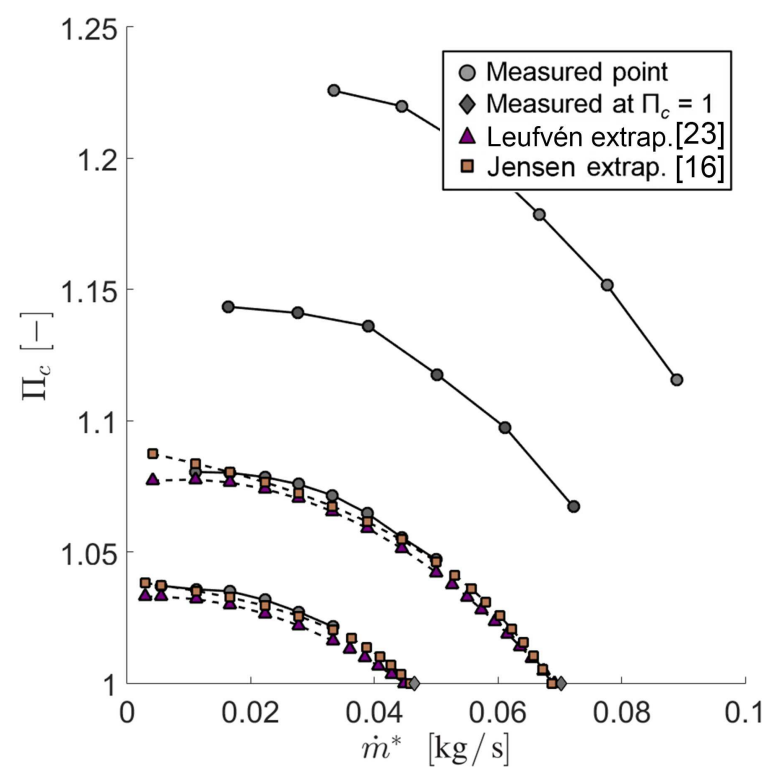

(a) Compressor $\mathrm{A}$

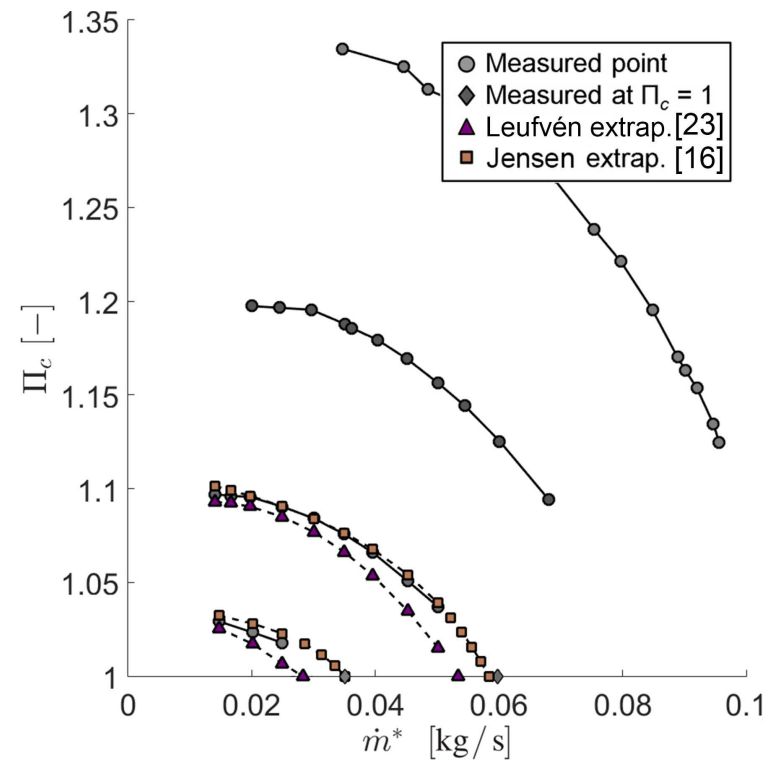

(b) Compressor B

Figure 6: Comparison of LS-extrapolating methods

\subsection{Region merging and additional features}

Once a model is selected for each region and the extrapolation is performed, a process is needed to couple the different extrapolated regions with 


\begin{tabular}{|l|c|c|}
\hline & Comp.A & Comp.B \\
\hline Iso-speed & $\epsilon$ Leufvén & $\epsilon$ Leufvén \\
\hline & {$[\%]$} & {$[\%]$} \\
\hline $\mathbf{1}$ & 1.1 & 7.11 \\
\hline
\end{tabular}

Table 5: High speed extrapolation comparison (error in $\left.\Pi_{c}=1\right)$.

the measured zone in order to produce a smooth continuous map to be used properly by the 1D gas dynamic code. Other additional aspects need to be dealt with in this last part of the approach.

\subsubsection{Linear blending}

Since this whole process is conducted to extend the operating range of 0D1D simulations, but without giving away the information that was already available in the measured data, a transition between the measured and the extrapolation region is needed in order to avoid having discontinuities and non-physical overshoots. For this purpose, two curves are blended using a linear weight factor. On one side, a spline is generated using the extrapolated point at $\Pi_{c}=1$ and two measured points with the highest mass flow of each iso-speed line. On the other side, the extrapolated line is taken into account. At this point, the weight factor of the spline takes a value of 1 for the lowest mass flow of each iso-speed line. As the compression ratio decreases and the mass flow increases, the weight factor of the spline goes to 0 and the extrapolated line rises to 1. An example of this process is shown in Fig. 8.

\subsubsection{Surge extrapolation}

Another consideration needed consists in the surge limit extrapolation, needed by the 0D-1D codes to set a closed stable region. This limit is defined by the lower mass flow rate points of each iso-speed line. The high and low speeds extrapolated lines are rearranged so the first points of each are on the predicted limit.

For low speeds, the surge margin is defined as a straight line which starts at zero corrected mass flow and $\Pi_{c}=1$. Then it is taken the lowest speed surge point to define the second point that will generate the surge limit. So, since the low speed extrapolated lines start from zero corrected mass flow, the points on the left of the surge limit are removed. 


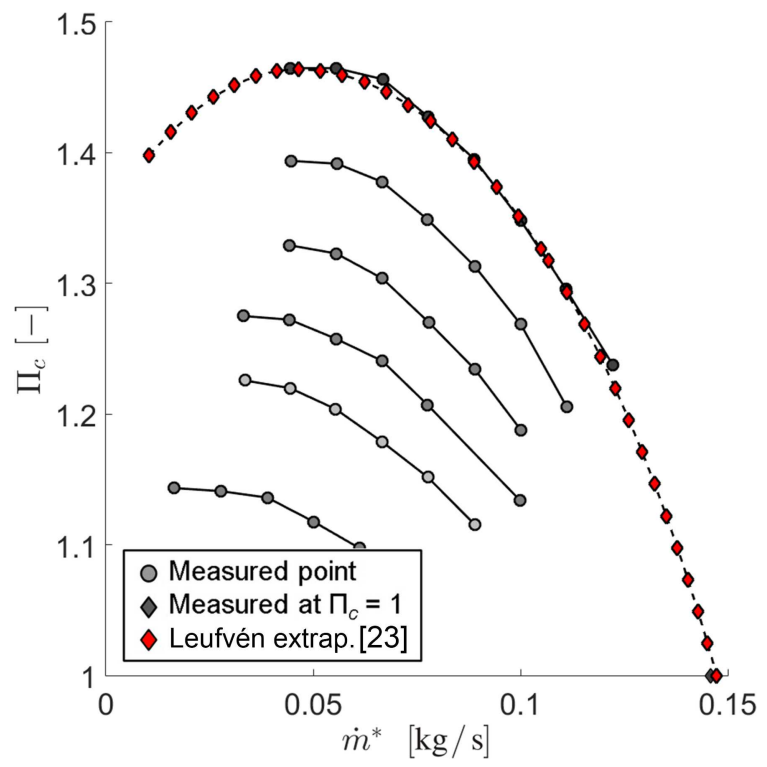

(a) Compressor $\mathrm{A}$

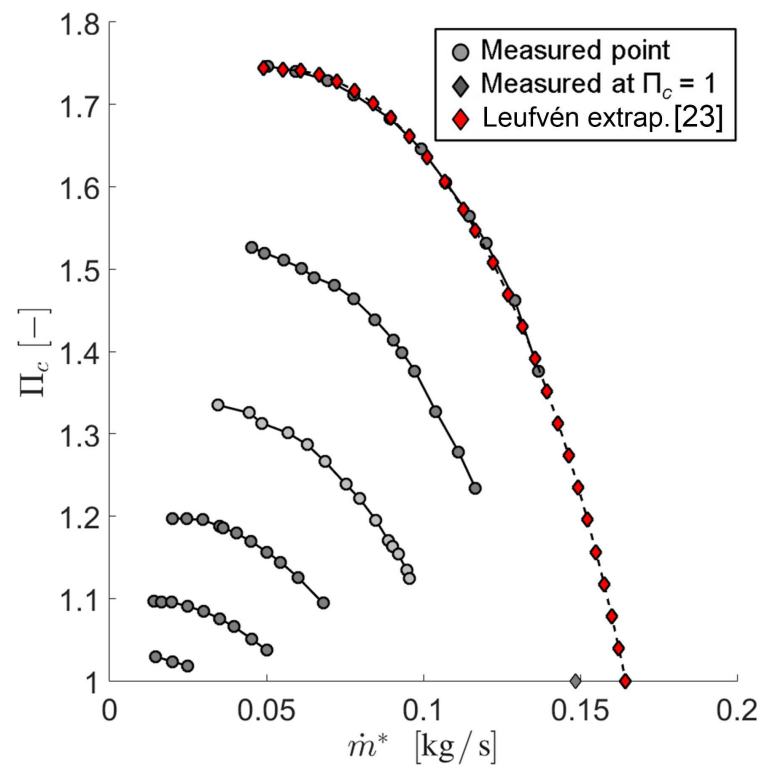

(b) Compressor B

Figure 7: HS-extrapolation

A different approach is needed for high speeds because Leufvén's extrapolated high speed line starts from the zero slope part of the line, and, fre- 


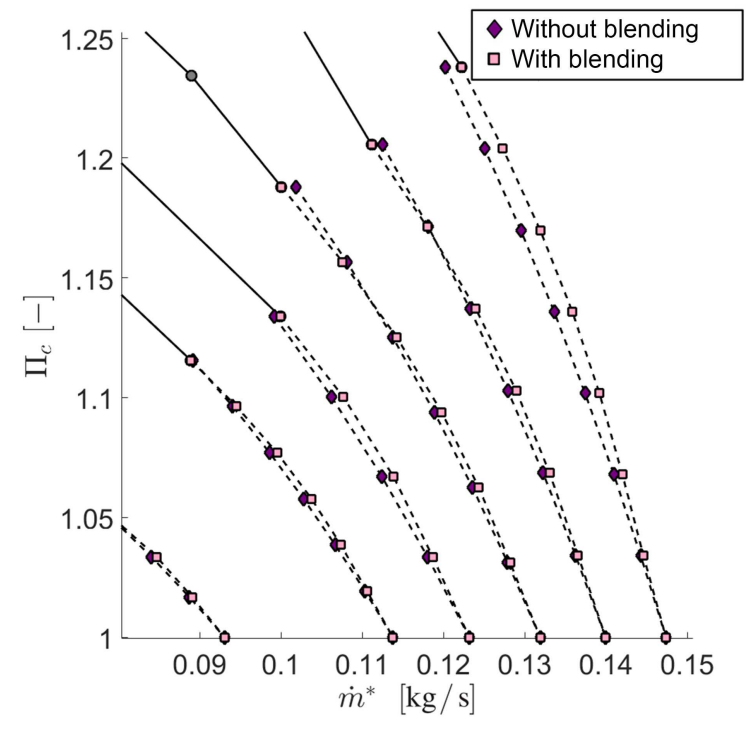

Figure 8: Linear blending processing

quently, this part falls far to the right of the surge limit and the positive slope region needs to be extrapolated as well. Thus, the corrected mass flow and compression ratio of the surge limit ought to be predicted. First, a parabolic regression is calculated as a function of the compression ratio and compressor speed of all the surge points of the map. Then, known the desired speed to be extrapolated, the compression ratio of this point is predicted using the previous regression. Following the same process used for the low speed surge line extrapolation, a surge line is created using the zero corrected mass flow and $\Pi_{c}=1$ point and the lowest mass flow rate point of the highest measured speed. Finally the extrapolated surge point is obtained in the crossing of the surge line with the compression ratio calculated with the parabolic regression. This point can be seen in Fig. 9.

Two cases may occur depending on the relative location between the extrapolated surge point and the zero slope point. If the zero slope point of the extrapolated speed line falls in lower mass flow rates, this portion on the left side of the surge limit is removed. Conversely, if there is a gap between the surge point and the zero slope point, a positive slope region needs to be extrapolated. 


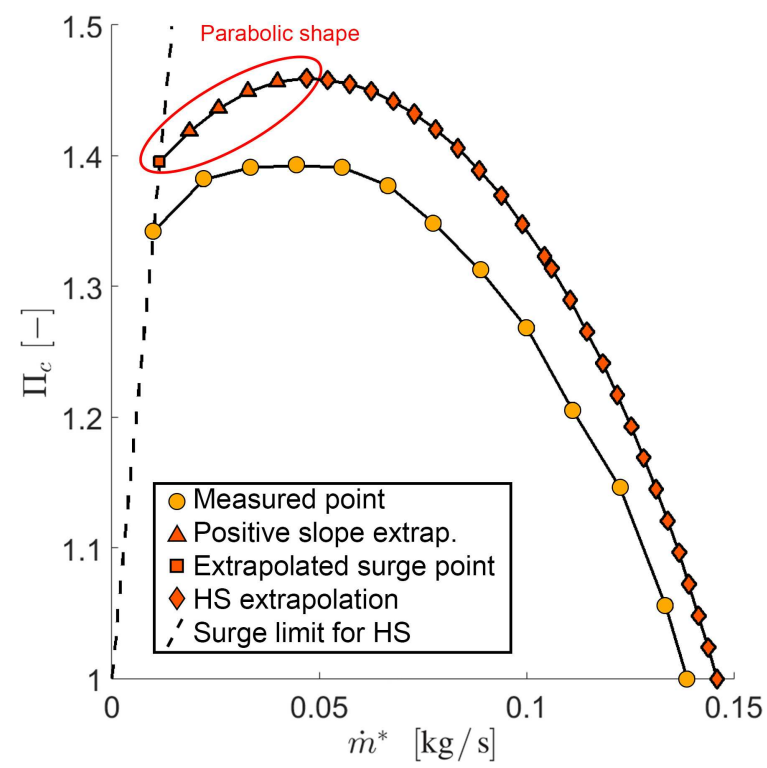

Figure 9: Surge limit and positive slope region extrapolation

\subsubsection{Positive slope region extrapolation}

After an extended exploration of different compressor maps, it was found that commonly, the positive slope part of the iso-speed lines could potentially be modelled with a polynomial curve of second order, i.e. a parabolic curve. The three known constraints that are needed to define a parabola are: the extrapolated surge point obtained previously, the zero slope point obtained with the Leufvén's model for the high speed line extrapolation and the condition of zero slope at that latter point. With this information, the parabolic curve is generated and added to the extrapolated line, as highlighted in Fig. 9. This is a simple, fast solution that provides a continuous and smooth line that will avoid convergence issues during the simulation of the 0D-1D code.

\subsubsection{Mapping}

Some additional features included in the final model are worth to be commented. The number of lines to extrapolate and the value of their speed is determined with the value of the measured lines. Thus, the mean separation between lines is kept for lower speeds, adding as many lines as possible. Regarding the high speed extrapolation, it is decided to add only one additional line. Since compressor manufacturers often measure up to already very high speeds, it makes no sense to add many lines. Furthermore, at high speeds 
the choke appearance leaves a narrow operating range. The discretization of the corrected mass flow rate is performed taken into account a similar discretization to the measured compressor map.

\section{Assessment of extrapolation approach}

Once the extrapolation approach is completely defined, the compressor maps used to compare and validate are tested. In Fig. 10(a) it is shown the comparison between the measured points and the extrapolated data. The errors found correspond mainly to the extrapolation of the positive slope region at high and low speeds. The LPR region extrapolation is quite accurate taking into account the difficulty of predicting the compressor behaviour near choke conditions. Regarding the extrapolation of compressor B shown in Fig. 10(b), a major error is found for the extrapolation of the points of $\Pi_{c}=1$ at high speeds. This issue is caused due to the small number of lines at high speed used to extrapolate.

Finally, two additional compressor maps corresponding to common automotive turbochargers are used to test the proposed model in its final form. Fig. 11(a) shows the extrapolation of compressor C. The measured points are depicted as circles while the extrapolated ones are depicted as triangles, squares and diamonds for the low pressure ratio, low speed and high speed extrapolation respectively. It can be observed the choke prediction as well as the positive slope region for the high speed extrapolated line.

Compressor D, depicted in Fig. 11(b), corresponds to a higher mass flow application. It is clearly seen the capabilities of the model to generate a complete map even if a small region of the operating range is measured, what represents a robust behaviour.

\section{Concluding remarks}

A compression ratio extrapolation method has been developed based on a zonal approach. Several models have been considered from the literature review, implemented and compared using two compressor maps with extreme off-design operating measurements. In each region a different comparison is made: enumerated below are the specific models selected for the proposed approach: 


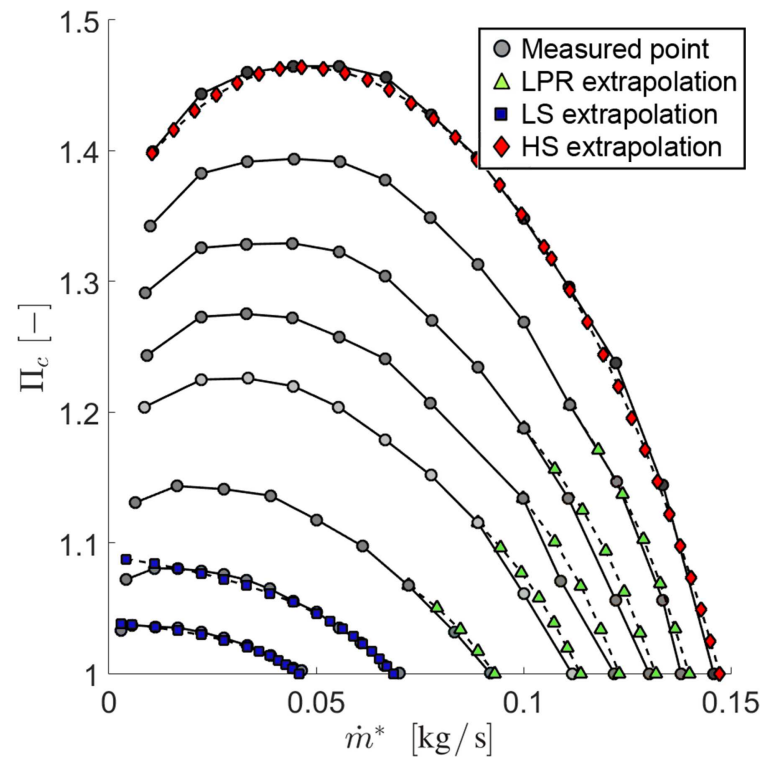

(a) Compressor $\mathrm{A}$

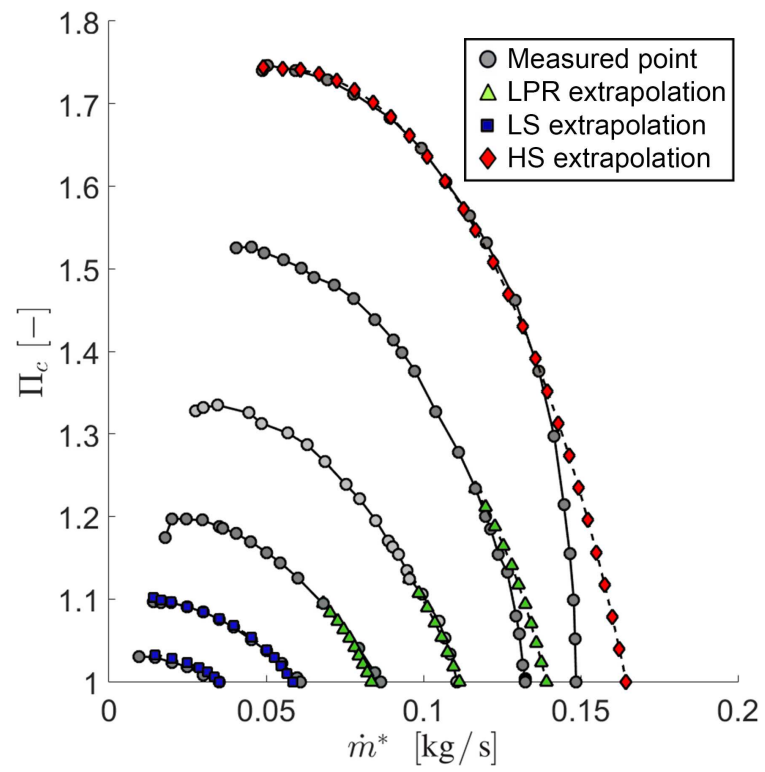

(b) Compressor B

Figure 10: Complete map extrapolation

- The low pressure ratio extrapolation is performed based on the ellipse model developed by Leufvén.[23] It is selected due to its higher accuracy 


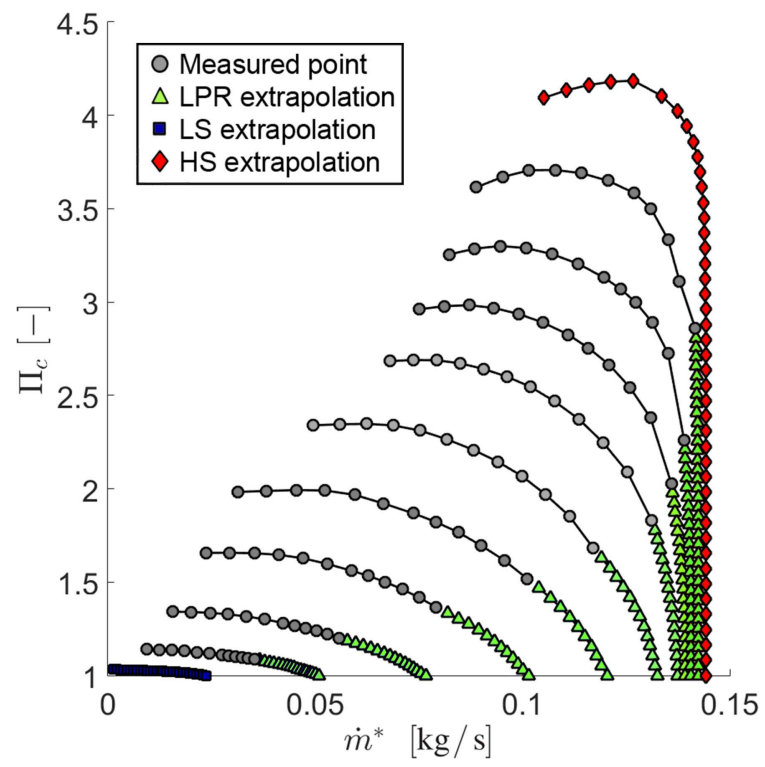

(a) Compressor $\mathrm{C}$

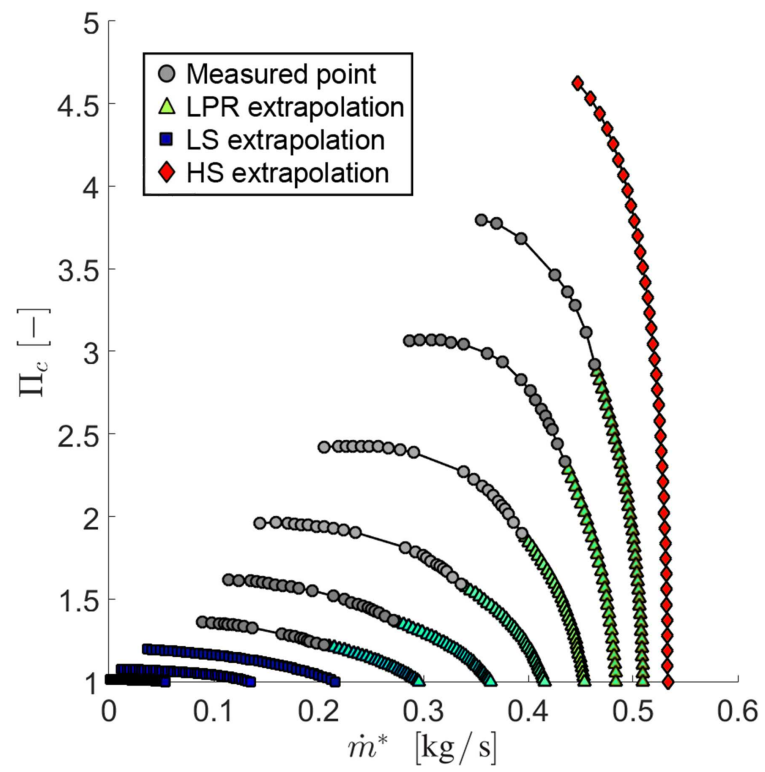

(b) Compressor D

Figure 11: Complete map extrapolation

and good prediction of the choke phenomenon. 
- At low speeds, the equation proposed by Jensen [16] shows the best prediction of the iso-speed lines.

- Finally, the high speed extrapolation is again performed based on the Leufvén's model.[23]

A region merging process and additional features have been introduced to allow a proper integration of different models as a simple compressor map, thus avoiding inconsistencies and discontinuities and leading to smooth maps which will ease the convergence of the 0D-1D gas dynamic simulations in which are interpolated. Two additional common compressor maps have been tested, resulting in satisfactory extrapolations. The developed approach, together with a method to extrapolate isentropic efficiency,[15] allow engine $1 \mathrm{D}$ codes to represent the compressor performance at all potential working conditions.

\section{Acknowledgements}

Daniel Tarí is partially supported through contract FPI-S2-2015-1095 of Programa de Apoyo para la Investigación y Desarrollo (PAID) of Universitat Politècnica de València.

\section{References}

[1] Mezher H, Chalet D, Migaud J et al. Wave dynamics measurement and characterization of a charge air cooler at the intake of an internal combustion engine with integration into a nonlinear code. Int $J$ Engine Res 2014; 15(6): 664-683.

[2] Lavoie G, Ortiz-Soto E, Babajimopoulos A et al. Thermodynamic sweet spot for highefficiency, dilute, boosted gasoline engines. Int $J$ Engine Res 2013; 14(3): 260-278.

[3] Dolz V, Novella R, García A et al. HD Diesel engine equipped with a bottoming Rankine cycle as a waste heat recovery system. Part 1: Study and analysis of the waste heat energy. Appl Therm Eng 2012; 36: 269-278. 


\section{List of Symbols}

$C$ Leufven's fitting constant -

$c_{p} \quad$ isobaric specific heat capacity $\mathrm{J} \cdot \mathrm{kg}^{-1} \cdot \mathrm{K}^{-1}$

$d$ diameter $\mathrm{m}$

$k \quad$ Jensen's fitting constant $\quad-$

$\dot{m}^{*} \quad$ corrected mass flow rate $\mathrm{kg} \cdot \mathrm{s}^{-1}$

$M \quad$ Mach number -

$N \quad$ compressor rotational speed rpm

$R \quad$ specific gas constant $\quad \mathrm{J} \cdot \mathrm{kg}^{-1} \cdot \mathrm{K}^{-1}$

$T$ temperature $\mathrm{K}$

$U$ blade tip speed $\mathrm{m} \cdot \mathrm{s}^{-1}$

$\Delta h$ enthalpy rise $\mathrm{J} \cdot \mathrm{kg}^{-1}$.

$\epsilon_{R} \quad$ relative error $\quad \%$

$\gamma \quad$ ratio of specific heats -

$\Pi$ compression ratio -

$\rho$ density $\mathrm{kg} \cdot \mathrm{m}^{-3}$

$\phi \quad$ flow coefficient -

$\psi$ load coefficient -

\section{Sub- and Superscripts}

1 inducer

2 exducer

c compressor related

is isentropic

\section{List of abbreviations}

0D zero dimensional

1D one dimensional

CFD computational fluid dynamics

HS high speed

ICE internal combustion engine

LPR low pressure ratio

LS low speed

[4] Serrano JR, Dolz V, Novella R et al. HD Diesel engine equipped with a bottoming Rankine cycle as a waste heat recovery system. Part 2: Evaluation of alternative solutions. Appl Therm Eng 2012; 36: 279- 
287.

[5] Bousquet Y, Carbonneau X, Dufour G et al. Analysis of the Unsteady Flow Field in a Centrifugal Compressor from Peak Efficiency to Near Stall with Full-Annulus Simulations. Int J Rotat Mach (Online) 2014; 2014: 11 .

[6] Broatch A, Galindo J, Navarro R et al. Numerical and experimental analysis of automotive turbocharger compressor aeroacoustics at different operating conditions. Int $J$ Heat Fluid Fl 2016; 61B: 245-255

[7] Semlitsch B and Mihaescu M. Flow phenomena leading to surge in a centrifugal compressor . Energy 2016; 103: 572-587.

[8] Hung KS, Chung JC, Liu CC et al. A study of off-design performance improvement for a centrifugal refrigerant compressor. Adv Mech Eng 2017; 9(3): 1-10.

[9] Leufvén O and Eriksson L. Measurement, analysis and modeling of centrifugal compressor flow for low pressure ratios. Int $J$ Engine Res $2016 ; 17(2): 153-168$.

[10] Serrano JR, Tiseira A, García-Cuevas LM et al. Radial turbine performance measurement under extreme off-design conditions. Energy 2017; 125: $72-84$.

[11] Serrano JR, Olmeda P, Tiseira A et al. Theoretical and experimental study of mechanical losses in automotive turbochargers. Energy 2013; 55: 888-898.

[12] Serrano JR, Olmeda P, Tiseira A et al. Importance of Mechanical Losses Modeling in the Performance Prediction of Radial Turbochargers under Pulsating Flow Conditions. SAE Int J Engines 2013; 6(2): 729-738.

[13] Galindo J, Tiseira A, Navarro R et al. Effect of the inlet geometry on performance, surge margin and noise emission of an automotive turbocharger compressor. Appl Therm Eng 2017; 110: 875-882.

[14] Eriksson L, Nezhadali V and Andersson C. Compressor Flow Extrapolation and Library Design for the Modelica Vehicle Propulsion Library - VehProLib. SAE Technical Paper 2016-01-1037, 2016. 
[15] Galindo J, Tiseira A, Navarro R et al. Compressor Efficiency Extrapolation for 0D-1D Engine Simulations. SAE Technical Paper 2016-01-0554, 2016.

[16] Jensen JP, Kristensen A, Sorenson S et al. Mean Value Modeling of a Small Turbocharged Diesel Engine. SAE Technical Paper 910070, 1991.

[17] Casey M and Robinson C. A method to estimate the performance map of a centrifugal compressor stage. J Turbomach 2013; 135(2): 021034.

[18] Gharaibeh K and Costall AW. A flow and loading coefficient-based compressor map interpolation technique for improved accuracy of turbocharged engine simulations. SAE Technical Paper 2017-24-0023, 2017.

[19] Dowell PG and Akehurst S. Advanced Mapping Techniques for Radial Compressor for Use in Real-Time Engine Models SAE Technical Paper 2010-01-1227, 2010.

[20] Martin G, Talon V, Higelin P et al. Implementing turbomachinery physics into data map-based turbocharger models. SAE Int. J. Engines 2009; 2(2009-01-0310): 211-229.

[21] El Hadef J. Approche quasi-systématique du contrôle de la chaîne d'air des moteurs suralimentés, basée sur la commande prédictive non linéaire explicite. PhD Thesis, Université d'Orléans, FR, 2014.

[22] Müller M, Hendricks E and Sorenson SC. Mean value modelling of turbocharged spark ignition engines. In International Congress $\&$ Exposition. SAE International. SAE Technical Paper 980784, 1998.

[23] Leufvén O. Modeling for control of centrifugal compressors. PhD Thesis, Linköping University, SE, 2013.

[24] Leufvén O and Eriksson L. Investigation of compressor correction quantities for automotive applications. Int J Engine Res 2012; 13(6): 588606.

[25] Leufvén O and Eriksson L. A surge and choke capable compressor flow model - Validation and extrapolation capability. Control Eng Pract 2013; 21(12): 1871-1883. 
[26] Serrano JR, Arnau FJ, Novella R et al. A Procedure to Achieve 1D Predictive Modeling of Turbochargers under Hot and Pulsating Flow Conditions at the Turbine Inlet. SAE Technical Paper 2014-01-1080, 2014.

[27] Serrano JR, Olmeda P, Arnau FJ et al. Analysis and Methodology to Characterize Heat Transfer Phenomena in Automotive Turbochargers. J Eng Gas Turb Power 2015; 137(2): 021901.

[28] Olmeda P, Tiseira A, Dolz V et al. Uncertainties in power computations in a turbocharger test bench. Measurement 2015; 59: 363-371. 